ACCEPTED MANUSCRIPT

\title{
Exfoliation of single layer BiTel flakes
}

To cite this article before publication: Bálint Fülöp et al 2018 2D Mater. in press https://doi.org/10.1088/2053-1583/aac652

\section{Manuscript version: Accepted Manuscript}

Accepted Manuscript is "the version of the article accepted for publication including all changes made as a result of the peer review process, and which may also include the addition to the article by IOP Publishing of a header, an article ID, a cover sheet and/or an 'Accepted Manuscript' watermark, but excluding any other editing, typesetting or other changes made by IOP Publishing and/or its licensors"

This Accepted Manuscript is @ 2018 IOP Publishing Ltd.

During the embargo period (the 12 month period from the publication of the Version of Record of this article), the Accepted Manuscript is fully protected by copyright and cannot be reused or reposted elsewhere.

As the Version of Record of this article is going to be / has been published on a subscription basis, this Accepted Manuscript is available for reuse under a CC BY-NC-ND 3.0 licence after the 12 month embargo period.

After the embargo period, everyone is permitted to use copy and redistribute this article for non-commercial purposes only, provided that they adhere to all the terms of the licence https://creativecommons.org/licences/by-nc-nd/3.0

Although reasonable endeavours have been taken to obtain all necessary permissions from third parties to include their copyrighted content within this article, their full citation and copyright line may not be present in this Accepted Manuscript version. Before using any content from this article, please refer to the Version of Record on IOPscience once published for full citation and copyright details, as permissions will likely be required. All third party content is fully copyright protected, unless specifically stated otherwise in the figure caption in the Version of Record.

View the article online for updates and enhancements. 


\title{
Exfoliation of single layer BiTeI flakes
}

\author{
Bálint Fülöp ${ }^{1,2}$, Zoltán Tajkov ${ }^{3}$, János Petô ${ }^{4}$, Péter Kun ${ }^{4}$, János \\ Koltai $^{3}$, László Oroszlány ${ }^{5}$, Endre Tóvári ${ }^{1,6}$, Hiroshi \\ Murakawa $^{7}$, Yoshinori Tokura ${ }^{8}$, Sándor Bordács ${ }^{1}$, Levente \\ Tapasztó $^{4}$, Szabolcs Csonka ${ }^{1,6}$ \\ ${ }^{1}$ Department of Physics, Budapest University of Technology and Economics, \\ Budafoki út 8, 1111 Budapest, Hungary. \\ ${ }^{2}$ MTA-BME Condensed Matter Research Group, Budafoki ut 8, 1111 Budapest, \\ Hungary. \\ ${ }^{3}$ Department of Biological Physics, Eötvös Loránd Univeŕsity, Budapest, Hungary. \\ ${ }^{4}$ Centre for Energy Research, Institute of Technical Physics and Materials Science, \\ 2D Nanoelectronics Lendület Research Group, Budapest, Hungary \\ ${ }^{5}$ Department of Physics of Complex Systems, Eötyös Loránd University, Budapest, \\ Hungary. \\ ${ }^{6}$ MTA-BME Lendület Nanoelectronics Research Group, Budafoki ut 8, 1111 \\ Budapest, Hungary. \\ ${ }^{7}$ Department of Physics, Osaka University, Toyonaka 560-0043, Japan. \\ ${ }^{8}$ Department of Applied Physics, The University of Tokyo, Tokyo 113-8656, Japan; \\ RIKEN Center for Emergent Matter Science (CEMS), Wako 351-0198, Japan.
}

\begin{abstract}
Spin orbit interaction is strongly enhanced in structures where a heavy element is embedded in an inversion asymmetric crystal field. A simple way for realizing such a setup is to take a single atomic layer of a heavy element and encapsulate it between two atomic layers of different elemental composition. BiTeI is a promising candidate for such a two dimensional crystal. In its bulk form BiTeI consists of loosely coupled three atom thick layers where a layer of high atomic number Bi are sandwiched between Te and I sheets. Despite considerable recent attention to bulk BiTeI due to its giant Rashba spin splitting, the isolation of a single layer remained elusive. In this work we report the first successful isolation and characterization of a single layer of BiTeI using a novel exfoliation technique on stripped gold. Our scanning probe studies and first principles calculations show that the fabricated $100 \mu \mathrm{m}$ sized BiTeI flakes are stable at ambient conditions. Giant Rashba splitting and spin-momentum locking of this new two dimensional crystal opens the way towards novel spintronic applications and synthetic topological heterostructures.
\end{abstract}

Keywords: Rashba spin splitting, BiTeI, stripped gold exfoliation, van der Waals heterostructures, topological insulator.

\section{1. Introduction}

37 Recently, the scientific interest and research activity regarding stacked two dimensional 38 (2D) van der Waals heterostructures have opened a new horizon to engineer materials 
at the nanoscale. These structures consist of single or few atomic layer thick crystals stacked on top of each other. The first member of the family of $2 \mathrm{D}$ materials was graphene, a zero-gap semiconductor, but it includes metals, semiconductors, insulators, furthermore semimetals, superconductors or strongly correlated materials [1,2]. For the application of these heterostructures in the field of spintronics and synthetic topological insulators, spin-momentum locking and band inversion are required, respectively, which can be provided by single or few-layer $2 \mathrm{D}$ crystals with high spin-orbit interaction (SOI) $[3,4]$. Crystals or nanostructures that lack inversion symmetry are good candidates to demonstrate strong SOI [5-8]. Among these, the polar semiconductor BiTeI stands out due to its giant Rashba splitting [9], but so far the fabrication of single layer BiTeI (SL BiTeI, one triplet of Te-Bi-I atomic layers) flakes has not been reported yet.

BiTeI is a member of a new class of polar crystals-with a layered structure, the class of ternary bismuth tellurohalides $\operatorname{BiTeX}(\mathrm{X}=\mathrm{I}, \mathrm{Br}, \mathrm{Cl})$, which recently attracted considerable attention [3,4,10-32]. The key component is $\mathrm{Bi}$, which as a heavy element has a strong atomic SOI. Its triangular lattice layer is stacked between a Te and an I (or $\mathrm{Br}$ or $\mathrm{Cl}$ ) layer (see figure 1a-b) [14]. The Bi layer along with Te forms a positively charged $(\mathrm{BiTe})^{+}$layer with similar geometry to metallic $\mathrm{Bi}$, whereas the I layer is negatively charged [20]. This polar structure along with the narrow band gap and the same orbital character of the bands at the top of the valence band and the bottom of the conduction band lead to the appearence of a giant Rashba spin splitting [10,11].

The Rashba effect results in a Hamiltonian $H_{\mathrm{R}}=\alpha \cdot \boldsymbol{\sigma}(\mathbf{n} \times \mathbf{k})$, where $\alpha$ is a coupling constant, $\boldsymbol{\sigma}$ is a vector of the Pauli matrices acting on the elecron spin, $\mathbf{n}$ is a unit vector perpendicular to the $2 \mathrm{D}$ plane of the crystal and $\mathbf{k}$ is the in-plane electron wave vector [33]. The corresponding band structure is shown at figure 1e. The Rashba effect dictates a spin-momentum locking as shown in the lower inset and induces an energy shift between opposite spin directions, which is described by the Rashba energy, $E_{\mathrm{R}} \propto \alpha^{2}$. $E_{\mathrm{R}}$ is exceptionally high for BiTeI: it is $E_{\mathrm{R}} \approx 110 \mathrm{meV}$ for the bulk crystal $[9,12]$. This is four times higher than the energy scale of room temperature thermal fluctuations $\left(k_{\mathrm{B}} T=25 \mathrm{meV}\right)$, two orders of magnitude higher than the spin splitting at a conventional InGaAs/InAlAs semiconductor interface, or on the surface of $\mathrm{Ag}(111)$ or $\mathrm{Au}(111)[6,14]$. Only extremely sensitive surface structures have been observed to produce higher Rashba energy [6], like Bi atoms on Ag surface existing only in ultra high vacuum. This built-in giant spin-orbit interaction makes BiTeI an attractive, novel component in van der Waals heterostructures.

Recently several theoretical works proposed the combination of BiTeI with other 2D crystals. According to first principles calculations of BiTeI/graphene [3,34] (and $\mathrm{BiTeCl}$ /graphene [35]) heterostructures, the strong Rashba interaction of BiTeI is expected to exert a significant influence on the Dirac electrons of graphene resulting in a nontrivial band structure, which paves the way for a new class of robust artificial topological insulators with several possible applications in spintronics, as demonstrated previously in the case of nanosheets [36-38]. It is also possible to combine SL BiTeI with topological insulators that host $2 \mathrm{D}$ helical Dirac states predicted to produce 


\section{Exfoliation of single layer BiTeI flakes}
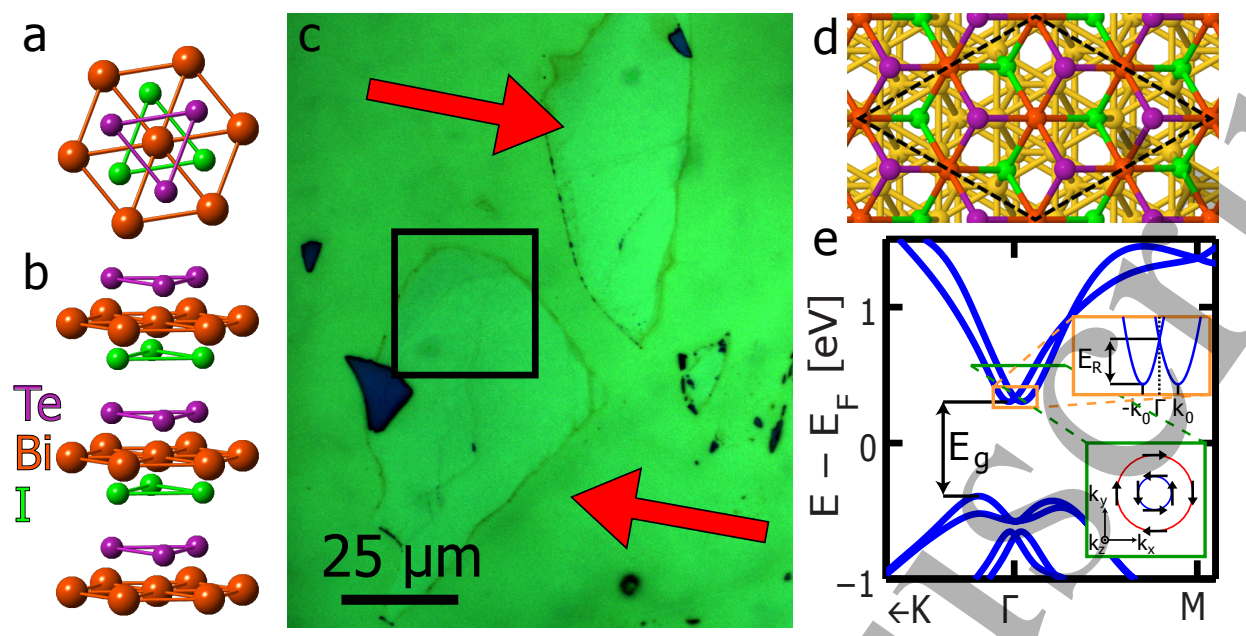

Figure 1. (a-b) Top and side view of the structure of the BiTeI crystals, respectively. The asymmetric stacking of the Te, Bi, I layers breaks the inversion symmetry and leads to the polar structure. (c) Channel-selective contrast enhanced optical micrograph of BiTeI flakes on a stripped gold surface after sonication. The green background corresponds to the Au substrate, the blue flakes are the thick BiTeI crystals. The light green patches with gray borders marked by red arrows are the SL BiTeI flakes under study in this work. The black square marks the location of the AFM measurements discussed in figure 4. (d) Illustration of the theoretically investigated BiTeI / Au system (I-faced). The unit cell is depicted with black dashed lines. (e) Dispersion relation (energy versus momentum relationship) in freestanding SL BiTeI along the $\mathrm{K}-\mathrm{\Gamma}-\mathrm{M}$ points. The band gap $E_{\mathrm{g}}$, the momentum offset $k_{0}$ and the Rashba Energy $E_{\mathrm{R}}$ are also indicated. Upper inset: zoom at the bottom of the conduction band with the relevant quantities indicated. Lower inset: schematic representation of the spin-momentum locking effect on the vertical cut of the dispersion relation along the marked green section.

more complex spin transport effects depending on the coupling strength between these systems [4]. Furthermore, a pair of inversely stacked SL BiTeI itself is also expected to exhibit topological insulating behaviour $[15,16]$. This shows the versatility of possible applications of SL BiTeI and the increasing demand for its production. However, no experimental demonstration of SL BiTeI has been reported yet. Previous experimental studies only focussed on bulk properties [11, 13, 14, 17, 20-29,32].

\section{Methods}

The standard mechanical exfoliation technique, using adhesive tape pressed onto and retracted from a $\mathrm{SiO}_{2}$ substrate, is successful in producing flakes of many $2 \mathrm{D}$ materials such as graphene and hexagonal boron nitride (hBN) because of the strong adhesion between these crystals and the $\mathrm{SiO}_{2}$ surface [39]. However, according to our early experience, in the case of BiTeI, the mechanical exfoliation results in only $50-100 \mathrm{~nm}$ thick flakes with lateral dimensions of a few micrometers and has very low yield. In the literature, BiTeI thin films with thicknesses between $70 \mathrm{~nm}$ and $10 \mu \mathrm{m}$ have been 
fabricated from polycrystalline powder using flash evaporation and their mechanical [40] and electrical [41] properties have been investigated, but these polycrystalline samples are far thicker than a single layer of the crystal.

In the current work SL BiTeI flakes were exfoliated with a novel method using freshly cleaved $\mathrm{Au}(111)$ substrate [42] that yields SL BiTeI flakes of lateral dimensions up to $100 \mu \mathrm{m}$. The underlying principle of the method is that the closest atomic layer to the Au substrate, be it either Te or I, forms stronger bonds to it than the cohesive energy between two BiTeI layers. Thus the last layer of BiTeI remains on the $\mathrm{Au}$ surface when a bulk BiTeI crystal comes into contact with it, while the rest of the crystal can be removed via sonication. To understand the exfoliation process we also performed calculations based on density functional theory (DFT) to obtain the geometry and binding energies. In the next sections we discuss the experimental and then the theoretical details of our work.

\subsection{Fabrication}

Gold layers of $100 \mathrm{~nm}$ thickness were grown epitaxially on mica prior to exfoliation. The exfoliation was performed on the Au surface obtained by cleaving the mica-Au interface, which consists of large area $\mathrm{Au}$ (111) facets. Bulk BiTeI crystals were grown by the Bridgman method as described in Ref. [14]. The crystals were characterized by various techniques: angle-resolved photoemission spectroscopy (ARPES), magnetic transport, optical spectroscopy and X-ray diffraction (XRD). Thick BiTeI flakes were prepared on scotch tape by consecutive folding several times and transferred on the $\mathrm{Au}$ (111) surface using a thermal release tape. After the removal of the thermal release tape by heating the sample on a hot plate up to $90^{\circ} \mathrm{C}$, the sample was mildly sonicated in room temperature acetone. The sonication causes some of the thick BiTeI flakes to separate from the substrate leaving only SL BiTeI pieces on the surface, which can be found using optical microscopy as detailed below.

\subsection{Optical and scanning probe microscopy}

A Zeiss Axio Imager optical microscope was used for the optical investigation of the samples. We took $2.5 \mathrm{x}$ mágnification pictures before and after the sonication and compared them to find areas where the bulk crystals detatched from the Au during the process (not shown in this paper). We found that the SL BiTeI flakes can be localized using channel-selective contrast-enhanced optical microscope images with a 100 microseope lens (see figure 1c). For the details of the image processing, see the supplementary material.

Seanning Tunneling Microscopy (STM) measurements were performed on a Nanoscope E instrument using standard Pt-Ir 90\%-10\% tips created by mechanical shearing. High resolution 2D maps were scanned at a current setpoint of $3 \mathrm{nA}$ with $5 \mathrm{mV}$ bias voltage, while large area $2 \mathrm{D}$ maps were scanned at $1 \mathrm{nA}$ and $200 \mathrm{mV}$. The differential conductance measurements were obtained by measuring I-V characteristics 


\section{Exfoliation of single layer BiTeI flakes}

at $1 \mathrm{nA}$ and $200 \mathrm{mV}$, then a numerical differentiation was applied to the average of dozens of individual measurements.

For Atomic Force Microscope (AFM) scans we used a Bruker Multimode 8 Nanocope V. AFM instrument in tapping mode. It has a larger scanning range than the STM device and sufficient resolution in the $\mathrm{z}$ axis to resolve atomic layer thicknesses. All STM and AFM measurements were performed under ambient conditions.

Raman measurements were performed using a Witec 300RSA + confocal Raman spectrometer with $532 \mathrm{~nm}$ excitation wavelength and a minimal spot size of approx. $0.5 \mu \mathrm{m}$.

\subsection{First principles calculations}

Density functional theory (DFT) calculations were performed for freestanding single layer samples and single layer slab of BiTeI placed on an $\mathrm{Au}$ substrate. For all cases the considered samples were separated with a minimum of $18.5 \AA$ thick vacuum in the perpendicular direction. For the sample on the substrate, a $2 \times 2$ supercell of SL BiTeI was placed on 6 layers of $3 \times 3$ supercell of (111) Au, thus the system consisted of 66 atoms in total: 54 gold atoms and 4 atoms of Bi, Te and I each. Both, Te and I facing substrate geometries were considered, i.e. $\mathrm{Au}^{6}$-Te-Bi-I, denoted as Te-faced, shown in figure $1 \mathrm{~d}$, and $\mathrm{Au}^{6}$-I-Bi-Te, denoted as I-faced.

Geometry optimization was performed by fixing the two Au plains furthest from the single layer and letting the remainder of the structure to relax. In the optimized geometry the substrate imposed a $1.3 \%$ increase on the BiTeI lattice constant, which although alters some calculated properties slightly, but is not expected to affect our qualitative conclusions.

The geometry optimization was calculated with both the projector augmentedwave method, as it is implemented in the VASP package [43,44], and with the linear combination of atomic orbitals method as it is implemented in the SIESTA package $[43,44]$. Both approaches resulted in essentially the same structure (see table 1).

The VASP code was further employed to calculate the binding energies and the STM image, while SIESTA calculations yielded species projected partial density of states (PDOS). The relaxed structure and binding energies were obtained neglecting spin-orbit coupling as these quantities are expected to be largely insensitive to it $[3,34]$.

In the VASP calculations a plane-wave cutoff of $500 \mathrm{eV}$ was used with a Brillouin zone sampling of a $12 \times 12 \times 1 \quad$ T-centered Monckhorst-Pack grid. Van der Waals interactions are taken into account through DFT-D3 Grimme corrections [45] for the Perdew-Burke-Ernzerhof (PBE) functionals [46].

The freestanding sample was relaxed without any restrictions while the sample on a substrate was constrained to the lattice of bulk Au. Relaxation was performed with a $3 \mathrm{meV} / \AA$ force tolerance. The STM image was simulated by calculating the local electron density of states (LDOS) according to the Tersoff-Hamann approach [47] using the VASP code. In figure $5 \mathrm{c}$ the LDOS was mapped in a $0.1 \mathrm{eV}$ energy window just 
Exfoliation of single layer BiTeI flakes

\begin{tabular}{c|cc|cc} 
& \multicolumn{2}{|c|}{ I-faced } & \multicolumn{2}{c}{ Te-faced } \\
& VASP $[\AA]$ & SIESTA $[\AA]$ & VASP $[\AA]$ & SIESTA $[\AA]$ \\
\hline lattice constant & 4.35 & 4.31 & 4.35 & 4.31 \\
\hline Bi-Te bond & 3.05 & 3.06 & $3.08 \pm 0.02$ & $3.10 \pm 0.02$ \\
Bi-I bond & $3.30 \pm 0.03$ & $3.28 \pm 0.02$ & $3.29 \pm 0.02$ & $3.26 \pm 0.01$ \\
\hline Au-Te bond & - & - & 2.81 & 2.92 \\
Au-I bond & 3.09 & 3.04 & - & -
\end{tabular}

Table 1. Summary of the geometrical parameters calculated with VASP and SIESTA. The SL-BiTeI was placed on top of 6 layers of $\mathrm{Au}(111)$ both in Te- and I-faced configurations. The lattice constant was fixed to the bulk value of $\mathrm{Au}$ calculated within the same method. There is a small alteration in the bond lengths due to the buckling on the gold surface. The parameters obtained by the two methods are in reasonable agreement.

below the Fermi energy and ca. $1 \AA$ above the surface according to common practice. Binding energies are obtained by comparing total energies of fully relaxed calculations of separated fractions and joined structures. This approach yields $57 \mathrm{meV} /$ atom binding energy for the case of graphite layers in good agreement with experimental results of $62 \pm 5 \mathrm{meV} /$ atom [48].

SIESTA results were obtained with a mesh cutoff of $300 \mathrm{Ry}$ with a $5 \times 5 \times 2 \quad \Gamma$ centered Monckhorst-Pack grid in the Brillouin zone. The force tolerance of relaxation was $20 \mathrm{meV} / \AA$ İn the Self-consistent calculations we employed the PBE functional and the pseudopotentials optimized by Rivero et al. [49] with a double-zeta polarized basis set. After relaxation a self-consistent single-point calculation was done with SOI included [50,51]. The sisl tool [52] was used to extract the PDOS from SIESTA calculations, sampling the Brillouin zone with a $70 \times 70 \times 1 \quad k$-points set.

\section{Results and Discussion}

In this section we focus on optical studies, and STM and AFM measurements performed on one representative SL BiTeI flake, then discuss them in light of our theoretical calculations. We produced in total 3 stripped gold samples using BiTeI crystals of two different crystal growth. In each case the total estimated area of the crystals pressed on the substrate is on the order of $1 \mathrm{~mm}^{2}$. The size of the substrates was approximately $4 \mathrm{~mm} \times 4 \mathrm{~mm}$. The exfoliation process works very efficiently: on all stripped gold chips one could easily find 3-5 areas with SL BiTeI of remarkable sizes of 10-100 $\mu \mathrm{m}$ (see the supplementary material).

The contour of the BiTeI flakes are recognizable on bare optical microscopy images, however a channel-selective contrast enhancement process allows a much better identification (see details in the supplementary material). A typical optical micrograph of a sample surface after exfoliation is shown in figure 1c, where the Au substrate is green, while thick BiTeI crystals that stayed on the surface after the sonication are blue 

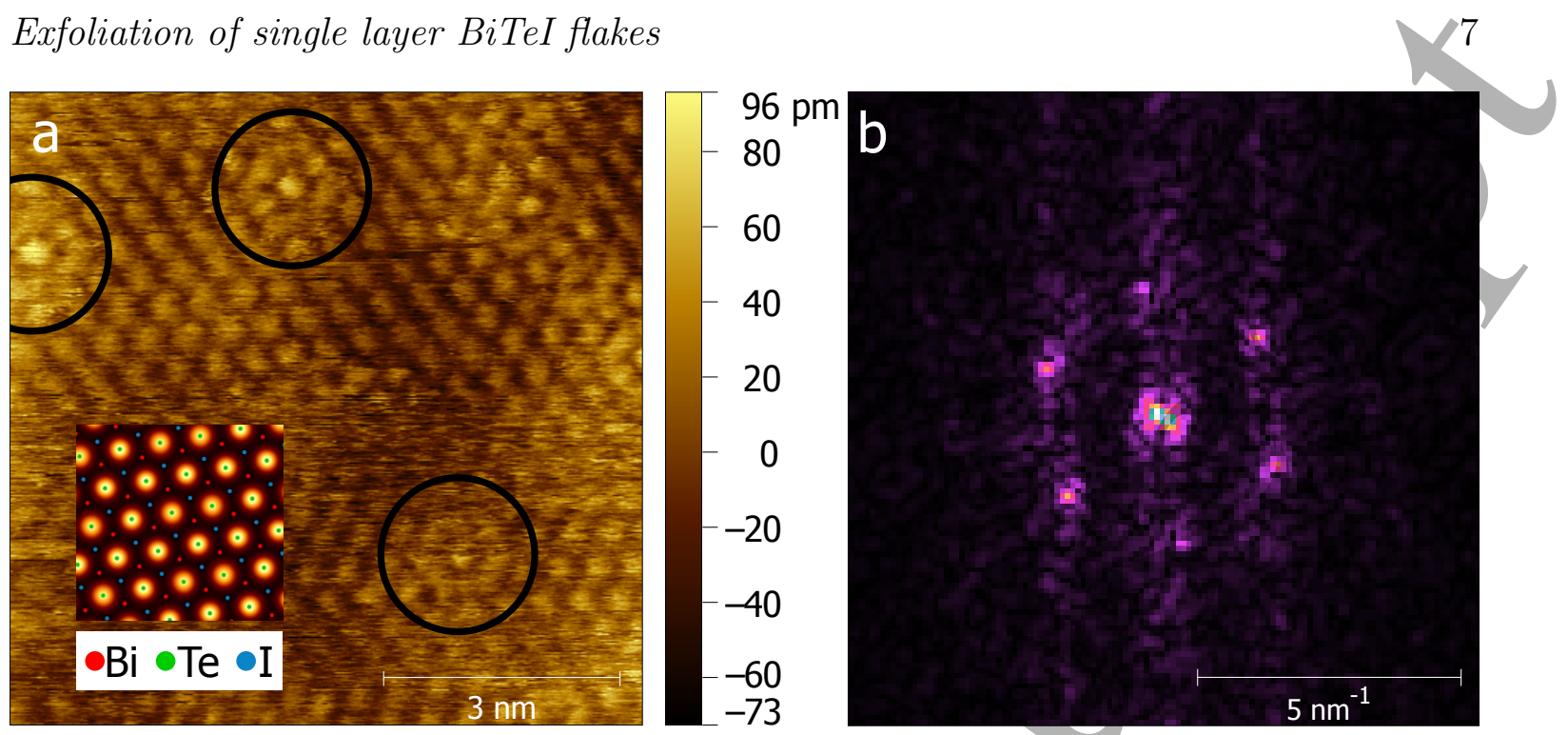

Figure 2. (a) STM measurement image of the atomic structure of the SL BiTeI flake in a $8 \mathrm{~nm} \times 8 \mathrm{~nm}$ window. The observed trigonal pattern corresponds to the crystal structure of the BiTeI. Black circles mark defect sites. Inset: simulated STM image of the Te-faced configuration of the same scale for comparison. The locations of the constituent atoms are marked by red, green and blue dots for Bi, Te and I, respectively. The simulated lattice matches remarkably well with the measurement. Note that the STM is sensitive to the location of the Te atoms albeit the I and Bi atoms are closer to the tip, because the highest PDOS belongs to Te at the Fermi energy. This behaviour is in agreement with our calculations presented in figure 5. (b) 2D FFT spectrum of the scan in (a). The peak positions correspond to $4.2 \AA \pm 0.2 \AA$, which is in agreement with the measured bulk lattice constants of BiTeI in Refs. $[10,53]$ and our calculated values, see table 1 .

due to the channel-selective contrast enhancement. Some of the thick BiTeI crystals that detached during sonication leave behind light green patches of several tens of microns lateral size, like the pair marked by red arrows. In the following we focus on these regions and show that SL BiTeI covers almost continuously these patches.

Atomic resolution STM measurements were made on these patches. Room temperature, ambient STM measurements revealed a trigonal atomic pattern at the surface (see figure 2a), similar to the bulk crystal structure of BiTeI. To precisely measure the periodicity of the observêd trigonal pattern, we performed a two dimensional Fourier transform (see figure $2 \mathrm{~b}$ ) and measured the positions of the maxima yielding a periodicity of $4.2 \pm 0.2 \AA$. This is in agreement with the in-plane lattice parameter of our calculations for the SL BiTeI on top of $\mathrm{Au}$, see table 1, and also with the measured bulk lattice parameter of BiTeI in the layer plane according to previous reports $(4.3 \AA[10,53])$. Furthermore it is significantly different from the gold lattice parameter in the (111) direction (2.9 $\AA$ [54]). Analyzing further figure $2 \mathrm{a}$ by comparing the measurement with the simulated STM map in the inset, one can find a remarkable match. Thus, one can conclude that after sonication BiTeI is still present on the substrate. Point defects are also visible on the image, as highlighted by black circles. By comparing them with defect states of bulk BiTeI [30,31], due to the absence of pronounced threefold symmetry and 


\section{Exfoliation of single layer BiTeI flakes}

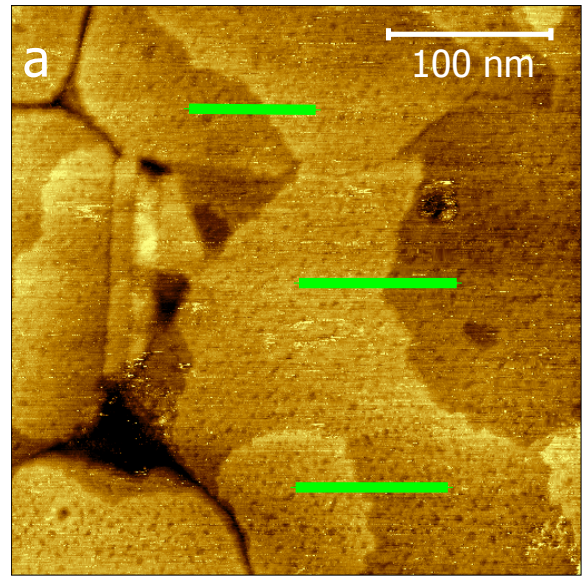

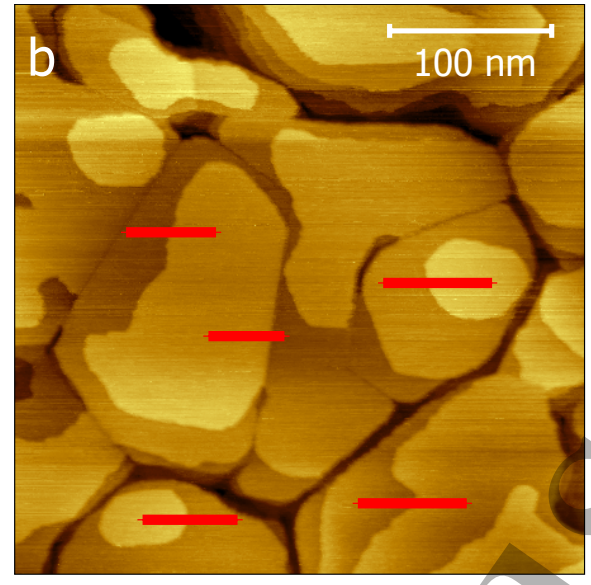

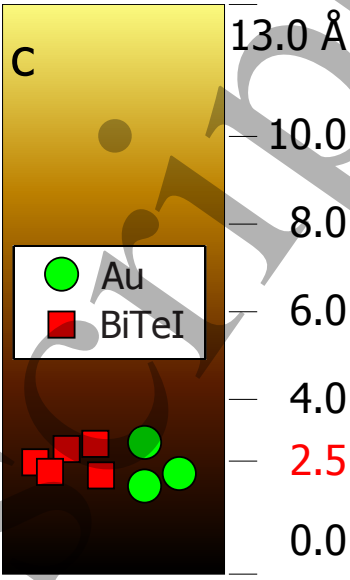

Figure 3. Comparison of surface topologies. (a), (b) STM image of a typical pure $\mathrm{Au}$ (111) and BiTeI-covered surface, respectively. The presence of BiTeI alters the surface significantly. The steps are more pronounced while the terraces are smaller in lateral extent leading to more steps present on the same scan size. Scan size is $350 \mathrm{~nm} \times 350 \mathrm{~nm}$. The green and red segments mark cuts along which step height measurements were performed; these are indicated on the heat map. (c) Heat map used on the maps (a-b) and marks of step heights measured along the marked segments on panel (a) (green) and panel (b) (red). The step heights measured on the BiTeI cloudy pattern do not differ from those measured on pure gold. The STM images were taken at ambient conditions.

the small height of $<20 \mathrm{pm}$ these defects resemble mostly antisites. We expect the crystal lattice apart from these point defects to be intact and the distribution of the elements uniform.

When zooming out for larger area scans, we found that the surface topology of the SL BiTeI flakes is also different from that of the pure Au substrate on the lengthscale of $1 \mathrm{\mu m}$ (see figure 3). The Au surfaces are well known to consist of large (111) terraces separated by single or multiple steps along (112) or (110) directions measuring $2.5 \AA$ step heights (see figure 3a) $[55,56]$. The SL BiTeI flakes show a characteristically different landscape (see figure 3b), which we call "cloudy" texture in the following. In this pattern one can find distinctive terraces of atomically flat regions similar to that of $\mathrm{Au}(111)$ faces, but in this case the boundaries of the terraces are more ragged, their size is smaller, and often multiple terraces occur on top of each other which is not the case for $\mathrm{Au}$. The two surface structures are also well distinguishable in larger scan ranges as a later AFM image shows (see figure $4 \mathrm{~b}$ ). The trigonal atomic structure presented in figure $2 \mathrm{a}$ can be generally found anywhere where the cloudy pattern is visible, but not in areas without it.

In order to identify whether the terraces are related to atomic steps of BiTeI or to the substrate, we measured the height of dozens of the terraces using linecuts in the scan direction, some of which are marked in figure 3a-b using green and red lines for the clean $\mathrm{Au}$ and the BiTeI-covered surfaces, respectively. The corresponding step heights arê indicated by markers on the heat map in figure 3c. We found that the measured step 


\section{Exfoliation of single layer BiTeI flakes}

height of the cloudy pattern corresponds to that of the $\mathrm{Au}(111)$ steps of $2.5 \AA[55,56]$, and is significantly smaller than that expected of bulk $\operatorname{BiTeI}(6.5 \AA[10])$. This suggests that these steps do not mark the border of BiTeI monolayers but the gold terraces of the surface that the BiTeI layer follows closely. To identify the thickness of the covering BiTeI layer we have to search for an area where the flake ends on the optical image or the cloudy pattern ends in STM (called "border regions").

In figure 4a we show an AFM overview image of the investigated flake depicted in figure 1a. The bright curved line highlighted by the two red arrows is the border of the flake, i.e. the top-left region is the Au surface whereas the larger part of the image bounded by the bright line is covered with BiTeI. At the border of the flake a thick and broad strip of accumulated material can be found. The width of this line is rather large, $2-4 \mu \mathrm{m}$, which is in the same length scale as the surface roughness of the Au substrate, thus the measurement of the layer thickness across the border can not be performed reliably. Therefore we looked for holes in the flake such as the one marked by the green square. In these holes the surface is deeper and the texture is different.

After zooming in on the area marked by the green square (figure 4b) one can recognize the cloudy pattern of BiTeI (same as in STM measurement in figure 3b) in the outer region whereas in the hole the original Au surface is present, which corroborates the visual impression that the BiTeI layer is missing in the hole. On the border of these holes the accumulated contamination is not present, therefore it is possible to zoom in further (see figure 4c) and measure directly the step height at the border (figure 4d). We investigated the step heights at various positions around the border of the hole, measuring a couple of linecuts using various PID control parameters, e.g. as seen in figure $4 \mathrm{c}-\mathrm{d}$. The measured step heights are in the range of $8.5 \pm 1.2 \AA$, which is close to the bulk lattice parameters of BiTeI in the out-of-plane direction $(6.5 \AA[10]$ or $6.8 \AA[53])$. Thus we conclude that the measured step height corresponds to a single layer step, and the regions showing the cloudy pattern are indeed covered with a SL BiTeI crystal. The small mismatch of the measured height and the lattice parameter can be attributed to the fact that height measurement on different substrates could deviate a few Angstroms in AFM profiles [57]. Thus, our findings indicate that SL BiTeI can indeed be separated by stripped gold exfoliation technique, making it a powerful method to produce large size SL 2D crystals from materials beyond graphene and transition metal dichalcogenides (TMDCs) [42].

We analyzed the BiTeI-covered surface a second time, several weeks after the exfoliation: the atomic structure of the BiTeI layer (as in figure 2) was still present which shows the long term environmental stability of the BiTeI monolayer. This finding is remarkable, since TMDCs containing Te are usually unstable in ambient conditions on the Au surface in the time scale of several hours according to our previous experiments. In the absence of degradation in air, a line of contamination on a flake edge can be attributed to the fabrication process: glue residues likely accumulate at the edges of a bulk flake, which is also visible in optical microscope as shown in figure 1c and makes the SL BiTeI flakes more visible despite the small color difference of the flake and the 
Exfoliation of single layer BiTeI flakes
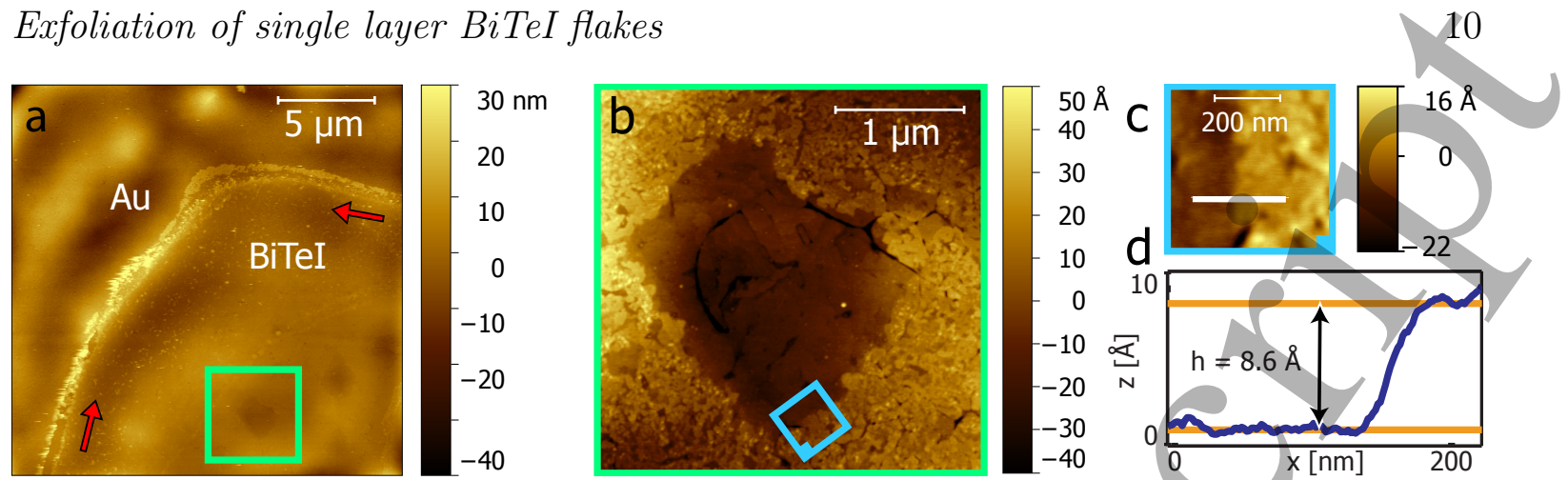

Figure 4. (a)AFM image of the location marked by the black square in figure 1c. The bright curved line marked by the red arrows is the top-left border of the flake. The green square marks one of the holes where the BiTeI layer is missing and the pure stripped gold surface is reproduced, presented in panel (b). For the details of the post-processing of the image, cf. the supplementary material. (b) In the outer region the cloudy pattern is visible which is characteristic of the BiTeI surfaces, whereas the inner region shows a landscape typical for a stripped gold surface. The blue square marks the location of the layer thickness measurement shown in (c-d). (c) Example of the layer thickness measurements and (d) the line plot of the corresponding cut.

substrate. Whereas a hole with clean edges is probably the result of tearing of the sheet along a grain boundary when the bulk crystal separates.

To further characterize the samples, we performed Raman measurements on the SL BiTeI areas, which did not result in a measurable signal, albeit we obtained a Raman signal on bulk BiTeI crystals similar to that reported in Ref. [58,59]. Note that a lack of Raman response was observed recently on $2 \mathrm{D}$ crystals with similar composition, e.g. on single layer $\mathrm{Bi}_{2} \mathrm{Te}_{3}$ as well [42].

To support the experimental results, we performed first principles calculations. First, we investigated the electronic structure of the freestanding single layer and compared it to the calculated properties of the bulk reproduced from Ref. [9]. The calculated band structure, depicted in figure 1e is characterized by a band gap of $E_{\mathrm{g}}=740 \mathrm{meV}$ and a Rasha energy $E_{\mathrm{R}}=35 \mathrm{meV}$, the former larger the latter smaller than their bulk value as expected.

As a next step, SL BiTeI on an Au surface was investigated (see figure 1d). Geometry optimization left the Au surface largely unaltered, while it introduced a small buckling in the BiTeI layer, as seen in table 1 . The binding energies of the relevant bonds are listed in table 2. The energy relations clearly indicate that both the Te-faced and the I-faced BiTeI binds stronger to the Au surface than to another SL BiTeI (see the first 3 rows), which is in agreement with the experimental findings that a SL BiTeI remains on the Au surface after sonication. As a reference, we also included the bonding energies between the constituents of a SL BiTeI which are much higher than the previous ones. Thus, it is highly unlikely that the SL BiTeI can be cleaved between Bi-Te or Bi-I planes, leaving only a part of the single layer on the substrate.

This result further supports that the multiple terraces at the cloudy pattern (see figure $3 b$ ) are not related to BiTeI but the underlying gold surface. On the other hand, 
Exfoliation of single layer BiTeI flakes

\begin{tabular}{rl} 
Bond & Binding energy \\
\hline $\mathrm{Au}^{6}-\mathrm{I}-\mathrm{Bi}-\mathrm{Te} \rightarrow \mathrm{Au}^{6}+\mathrm{BiTeI}$ & $681 \mathrm{meV}$ \\
$\mathrm{Au}^{6}-\mathrm{Te}-\mathrm{Bi}-\mathrm{I} \rightarrow \mathrm{Au}^{6}+\mathrm{BiTeI}$ & $969 \mathrm{meV}$ \\
$\mathrm{BiTeI}-\mathrm{BiTeI} \rightarrow \mathrm{BiTeI}+\mathrm{BiTeI}$ & $543 \mathrm{meV}$ \\
$\mathrm{Te}-\mathrm{Bi}-\mathrm{I} \rightarrow \mathrm{Te}-\mathrm{Bi}+\mathrm{I}$ & $2.74 \mathrm{eV}$ \\
$\mathrm{I}-\mathrm{Bi}-\mathrm{Te} \rightarrow \mathrm{I}-\mathrm{Bi}+\mathrm{Te}$ & $3.64 \mathrm{eV}$
\end{tabular}

Table 2. Binding energies calculated by PBE + Grimme method as implemented in the VASP package. The values represent the energy needed to break the bond as marked by the arrow. The results indicate that the BiTeI tends to stick to the Au surface stronger than to another layer of BiTeI, either Te or I-faced. The last two rows demonstrate that the internal bonds are much stronger than the interlayer binding energies, making it unlikely to leave only part of the BiTeI layer on the substrate.

the strong adhesion between the BiTeI and the Au substrate can also be a reason why the surface structure of the $\mathrm{Au}$ is significantly different under BiTeI coverage (cloudy pattern): the strong bonds can pin the Au atoms which/otherwise have high surface mobility; and also force the BiTeI to follow the terraces. This surface reconstruction is likely to be induced by the relative high temperature $\left(T \approx 90 \mathrm{C}^{\circ}\right)$ that the sample was exposed to during fabrication.

In the following, we calculated the PDOS on the constituents with and without the presence of the $\mathrm{Au}$ substrate (see figure 5a-b, respectively). In the case of the freestanding SL BiTeI one can see a gap of $0.76 \mathrm{eV}$ where the PDOS is zero for all the components, therefore we expect the single layer to show insulating behavior in this regime (see figure $5 \mathrm{a}$ and figure $1 \mathrm{e}$ ). In the whole investigated range of $\pm 3 \mathrm{eV}$, the position of the most prominent peaks in the PDOS are very similar for Bi, Te and I. One can observe that in the negative energy range the Te and I have more contribution while the part of the Bi orbitals increases towards higher energies. In the middle range, between 1 and $2 \mathrm{eV}$, the Te has the highest PDOS.

The PDOS is altered once the sample is placed on top of the Au substrate (as the Te-faced case depicted in figure $5 \mathrm{~b}$ ). While the main features of the freestanding sample can still be identified, the main effect is the smearing of the bulk gap of the SL BiTeI due to hybridization of BiTeI with the Au atoms. Thus one can safely conclude that a SL BiTel flake on a stripped gold substrate is not expected to show insulating characteristics. The qualitative features of Te-faced and I-faced (not shown here) structures are largely the same.

We performed $\mathrm{dI} / \mathrm{dV}$ measurements on several different locations of the SL BiTeI flake and no band gap was found in agreement with the above calculation. A typical example of a $\mathrm{dI} / \mathrm{dV}$ curve measured on the cloudy pattern at ambient conditions is shown in figure $5 \mathrm{c}$, where the numerical derivative remains always positive. The applied bias voltage was limited to $|\mathrm{V}|<500 \mathrm{mV}$ since beyond this range deviation from the simple tunnel limit is expected at ambient conditions. Due to the high instability of the environment a considerable amount of noise appears as random peaks and irregularities 
Exfoliation of single layer BiTeI flakes

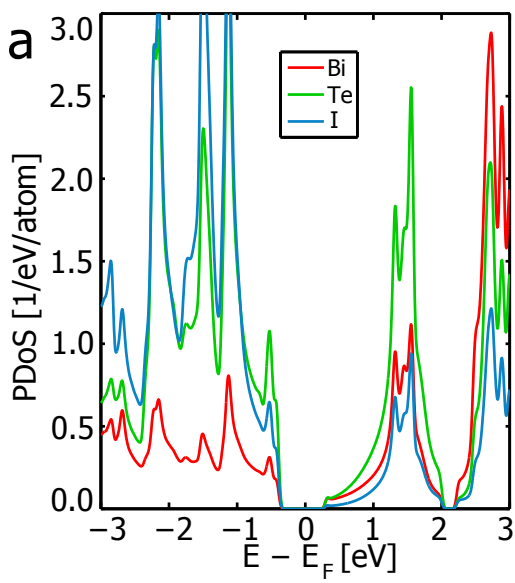

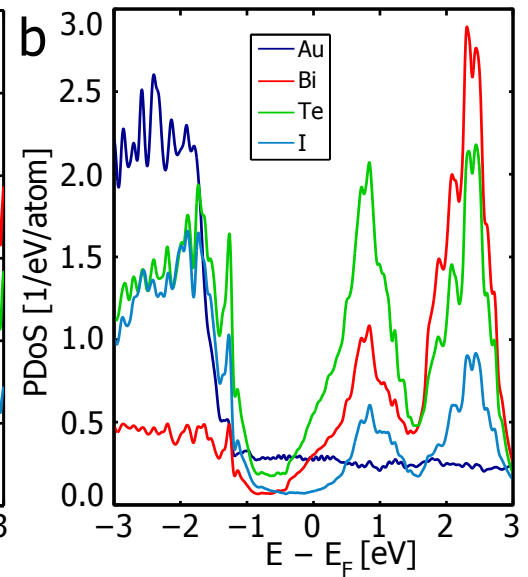

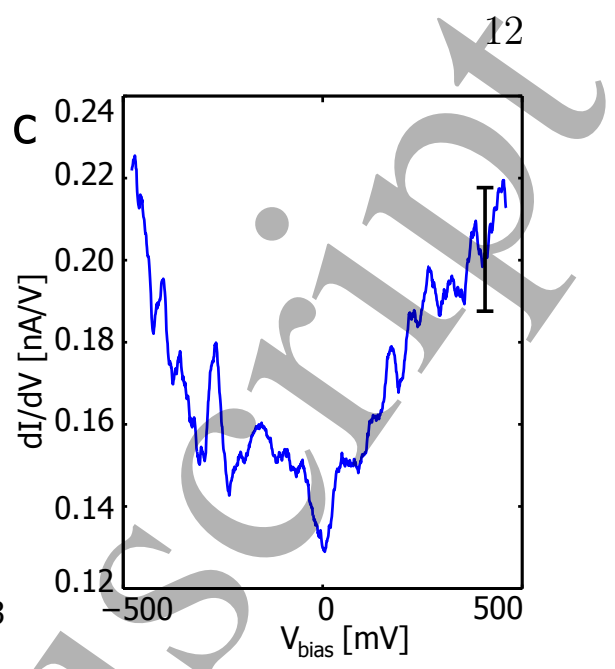

Figure 5. (a) Calculated partial density of states (PDOS) of the freestanding SL BiTeI. (b) PDOS of the SL BiTeI on Au, Te-faced (i.e. Au ${ }^{6}$-Te-Bi-I). Comparing (a) and (b) one can conclude that the presence of $\mathrm{Au}$ suppresses the presence of a band gap. (c) Numerical derivative of an I-V curve measured at ambient conditions on a SL BiTeI atomic structure and the characteristic error bar of $\mathrm{dI} / \mathrm{dV}$ values is shown. As a generic feature, the derivative never goes to zero, which is in agreement with the calculated PDOS curves of (b).

(see error bar) which are not related to any of the peaks in figure 5b. The non-zero $\mathrm{dI} / \mathrm{dV}$ value around zero bias and slight increase of $\mathrm{dI} / \mathrm{dV}$ towards finite bias voltage are the generic feature of the $\mathrm{dI} / \mathrm{dV}$ measurements one can read from the plot. This is in agreement with the calculated PDOS dominated by the hybridization of BiTeI and $\mathrm{Au}$.

\section{Conclusions}

We demonstrated for the first time that single layer flakes can be realized from the giant Rashba spin-orbit material BiTeI. The stripped gold exfoliation technique provides an efficient way to produce flakes with a size of $100 \mu \mathrm{m}$, which are stable at ambient conditions for at least several weeks. We showed that the position of the SL BiTeI flakes can be identified by simple optical microscopy. Atomic resolution STM measurements confirmed the presence of the SL BiTeI layer on the Au surface by resolving the in-plane crystal structure of BiTeI. AFM measurements showed that the flakes cover large areas continuously with only a few holes. Step height measurements across the edges of these holes confirmed that the flake thickness corresponds to SL BiTeI. Our first principles calculations also predict the formation of SL BiTeI due to the strong bonding between Te and I to Au substrate. Moreover, BiTeI strongly hybridizes with the Au substrate, which results in a finite DoS in the gap, in accordance with the differential conductance measurements. The first exfoliation of SL BiTeI adds a new member to the possible building blocks of van der Waals heterstructures. With its giant Rashba spin splitting, our results open the way to engineer novel 2D heterostructures with special spin-based functionality or topological protection. 
Exfoliation of single layer BiTeI flakes

\section{Acknowledgments}

This work was financially supported by the Flag-ERA iSpinText project (NN118996), the Flag-ERA Topograph project (NN 127903), the COST CA16218 Nanocohybri program, the "Lendület" program of the Hungarian Academy of Sciences and the Hungarian National Research, Development and Innovation Office (NKFIH) via the National Quantum Technologies Program NKP-2017-00001; grants no. K112918, K115608, K108676, FK124723 and K115575 for Z.T., J.K. and L.O.; grant no. K108753 for J.P., P.K. and L.T.; grant no. PD111756 for S.B.

L.O., J.K. and S.B. acknowledge the Bolyai program of the Hungarian Academy of Sciences. J.P., P.K. and L.T. acknowledge financial supportfrom ERC StG Nanofab2D

\section{References}

[1] A. K. Geim and I. V. Grigorieva, Nature 499(7459), 419-425 (2013).

[2] K. J. Koski and Y. Cui, ACS Nano 7(5), 3739-3743 (2013).

[3] L. Kou, S. C. Wu, C. Felser, T. Frauenheim, C. Chen, and B. Yan, ACS Nano 8(10), 10448-10454 (2014), PMID: 25226453.

[4] S. V. Eremeev, S. S. Tsirkin, I. A. Nechaev, P.M. Echenique, and E. V. Chulkov, Scientific Reports 5(August), 12819 (2015).

[5] S. LaShell, B. A. McDougall, and E. Jensen, Phys. Rev. Lett. 77(Oct), 3419-3422 (1996).

[6] C. R. Ast, J. Henk, A. Ernst, L. Moreschini, M. C. Falub, D. Pacilé, P. Bruno, K. Kern, and M. Grioni, Phys. Rev. Lett. 98(May), 186807 (2007).

[7] Y. M. Koroteev, G. Bihlmayer, J.E. Gayone, E. V. Chulkov, S. Blügel, P. M. Echenique, and P. Hofmann, Phys. Rev. Lett. 93(Jul), 046403 (2004).

[8] J. Nitta, T. Akazaki, H. Takayanagi, and T. Enoki, Phys. Rev. Lett. 78(Feb), 1335-1338 (1997).

[9] M. S. Bahramy, R. Arita, and N. Nagaosa, Phys. Rev. B 84(Jul), 041202 (2011).

[10] V. A. Kulbachinskii, V. G. Kytin, A. A. Kudryashov, A. N. Kuznetsov, and A. V. Shevelkov, Solid State Chemistry and Materials Science of Thermoelectric Materials 193(September), 154-160 (2012).

[11] C. Martin, A. V. Suslôv, S. Buvaev, A. F. Hebard, P. Bugnon, H. Berger, A. Magrez, and D. B. Tanner, EPL (Europhysics Letters) 116(5), 57003 (2016).

[12] M. Sakano, M. S. Bahramy, A. Katayama, T. Shimojima, H. Murakawa, Y. Kaneko, W. Malaeb, S. Shin, K. Ono, H. Kumigashira, R. Arita, N. Nagaosa, H. Y. Hwang, Y. Tokura, and K. Ishizaka, Phys. Rey. Lett. 110(Mar), 107204 (2013).

[13] G. Landolt, S. V. Eremeev, Y.M. Koroteev, B. Slomski, S. Muff, T. Neupert, M. Kobayashi, V.N. Strocov, T. Schmitt, Z.S. Aliev, M.B. Babanly, I. R. Amiraslanov, E. V. Chulkov, J. Osterwalder, and J.H. Dil, Phys. Rev. Lett. 109(Sep), 116403 (2012).

[14] K. Ishizaka, M. S. Bahramy, H. Murakawa, M. Sakano, T. Shimojima, T. Sonobe, K. Koizumi, S. Shin, H. Miyahara, A. Kimura, K. Miyamoto, T. Okuda, H. Namatame, M. Taniguchi, R. Arita, N. Nagaosa, K. Kobayashi, Y. Murakami, R. Kumai, Y. Kaneko, Y. Onose, and Y. Tokura, Nat Mater 10(7), 521-526 (2011).

[15] I. A. Nechaev, S. V. Eremeev, E. E. Krasovskii, P. M. Echenique, and E. V. Chulkov, Scientific Reports 7(March), 43666 (2017).

[16] S. V. Eremeev, I. A. Nechaev, and E. V. Chulkov, Phys. Rev. B 96(15), 155309 (2017).

[17] M. S. Bahramy, B. J. Yang, R. Arita, and N. Nagaosa, Nature Communications 3(February), 679 (2012).

[18] A. Ohmura, Y. Higuchi, T. Ochiai, M. Kanou, F. Ishikawa, S. Nakano, A. Nakayama, Y. Yamada, and T. Sasagawa, Phys. Rev. B 95(Mar), 125203 (2017). 
Exfoliation of single layer BiTeI flakes

[19] S. V. Eremeev, I. A. Nechaev, Y. M. Koroteev, P. M. Echenique, and E. V. Chulkov, Phys. Rev. Lett. 108(Jun), 246802 (2012).

[20] A. V. Shevelkov, E. V. Dikarev, R. V. Shpanchenko, and B. A. Popovkin, Journal of Solid State Chemistry 114(2), 379-384 (1995).

[21] M. Kanou and T. Sasagawa, Journal of Physics: Condensed Matter 25(13), 135801 (2013).

[22] M. Sakano, J. Miyawaki, A. Chainani, Y. Takata, T. Sonobe, T. Shimojima, M. Oura, S, Shin, M. S. Bahramy, R. Arita, N. Nagaosa, H. Murakawa, Y. Kaneko, Y. Tokura, and K. Ishizaka, Phys. Rev. B 86(Aug), 085204 (2012).

[23] B. Monserrat and D. Vanderbilt, Temperature dependence of the bulk rashba splitting in the bismuth tellurohalides, 2017.

[24] A. Crepaldi, L. Moreschini, G. Autès, C. Tournier-Colletta, S. Moser, N. Virk, H. Berger, P. Bugnon, Y. J. Chang, K. Kern, A. Bostwick, E. Rotenberg, O. V. Yazyev, and M. Grioni, Phys. Rev. Lett. 109(Aug), 096803 (2012).

[25] C. J. Butler, H. H. Yang, J. Y. Hong, S. H. Hsu, R. Sankar, C. I. Lu, H.Y. Lu, K. H. O. Yang, H. W. Shiu, C. H. Chen, C. C. Kaun, G. J. Shu, F. C. Chou, and M. T. Lin, NatComm 5(June), 4066 (2014).

[26] J. S. Lee, G. A. H. Schober, M. S. Bahramy, H. Murakawa, Y. Onosé, R. Arita, N. Nagaosa, and Y. Tokura, Phys. Rev. Lett. 107(Sep), 117401 (2011).

[27] L. Demkó, G. A. H. Schober, V. Kocsis, M. S. Bahramy, H. Murakawa, J. S. Lee, I. Kézsmárki, R. Arita, N. Nagaosa, and Y. Tokura, Phýs. Rev. Lett. 109(Oct), 167401 (2012).

[28] S. Bordács, J. G. Checkelsky, H. Murakawa, H. Y. Hwang, and Y. Tokura, Phys. Rev. Lett. 111(Oct), 166403 (2013).

[29] N. Ogawa, M. S. Bahramy, H. Murakawa, Y. Kaneko, and Y. Tokura, Phys. Rev. B 88(Jul), 035130 (2013).

[30] S. Fiedler, L. El-Kareh, S. V. Eremeev, O.E. Tereshchenko, C. Seibel, P. Lutz, K. A. Kokh, E. V. Chulkov, T. V. Kuznetsova, V. I. Grebennikoy, H. Bentmann, M. Bode, and F. Reinert, New Journal of Physics 16(7), 075013 (2014).

[31] Y. Kohsaka, M. Kanou, H. Takagi, T. Hanaguri, and T. Sasagawa, Phys. Rev. B 91(Jun), 245312 (2015).

[32] C. R. Wang, J. C. Tung, R. Sankar, C.T. Hsieh, Y. Y. Chien, G. Y. Guo, F. C. Chou, and W. L. Lee, Phys. Rev. B 88(Aug), 081104 (2013).

[33] Y. A. Bychkov and E. I. Rashba, Journal of Experimental and Theoretical Physics 39(2), 78-82 (1984).

[34] Z. Tajkov, D. Visontai, P. Rakyta, L. Oroszlány, and J. Koltai, Physica Status Solidi C pp. 1700215-n/a (2017), 1700215.

[35] S. V. Eremeev, I. A. Nechaev, P. M. Echenique, and E. V. Chulkov, Scientific Reports 4(November), 6900 (2014).

[36] N. Cheng, J. Tian, Q. Liu, C. Ge, A. H. Qusti, A. M. Asiri, A. O. Al-Youbi, and X. Sun, ACS Appl. Mater. Interfaces 5(15), 6815-6819 (2013).

[37] J. Tian, Q. Liu, A. M. Assiri, A. O. Al-Youbi, and X. Sun, Anal. Chem. 85(11), 5595-5599 (2013).

[38] J. Tian, Q. Liu, A. M. Asiri, K. A. Alamry, and X. Sun, ChemSusChem 7(8), 2125-2130 (2014).

[39] S. P. Koenig, N. G. Boddeti, M. L. Dunn, and J. S. Bunch, Nat Nano 6(9), 543-546 (2011).

[40] L. V. O. et al., Izv VUZ Fiz 11, 117-120 (1972).

[41] L.V. O. et al., Izv VUZ Fiz 11, 120-122 (1972).

[42] G. Z. Magda, J. Petô, G. Dobrik, C. Hwang, L.P. Biró, and L. Tapasztó, Scientific Reports $\mathbf{5}$ (October), 14714 (2015).

[43] G. Kresse and J. Hafner, Physical Review B 47(1), 558 (1993).

[44] G. Kresse and J. Furthmüller, Physical review B 54(16), 11169 (1996).

[45] S. Grimme, J. Antony, S. Ehrlich, and H. Krieg, The Journal of Chemical Physics 132(15), 154104 (2010).

[46] J. P. Perdew, K. Burke, and M. Ernzerhof, Phys. Rev. Lett. 77(Oct), 3865-3868 (1996). 


\section{Exfoliation of single layer BiTeI flakes}

454

[47] J. Tersoff and D. R. Hamann, Phys. Rev. B 31(2), 805-813 (1985).

[48] R. Zacharia, H. Ulbricht, and T. Hertel, Phys. Rev. B 69(Apr), 155406 (2004).

[49] P. Rivero, V. M. García-Suárez, D. Pereñiguez, K. Utt, Y. Yang, L. Bellaiche, K. Park, J. Ferrer, and S. Barraza-Lopez, Computational Materials Science 98(February), 372-389 (2015).

[50] D. Hobbs, G. Kresse, and J. Hafner, Phys. Rev. B 62(Nov), 11556-11570 (2000).

[51] V. M. García-Suárez, C. M. Newman, C. J. Lambert, J. M. Pruneda, and J. Ferrer, The European Physical Journal B 40(4), 371-377 (2004).

[52] N. R. Papior, sisl: v0.8.5.

[53] R. Sankar, I. Panneer Muthuselvam, C. J. Butler, S. C. Liou, B. H. Chen, M. W. Chu, W. L. Lee, M. T. Lin, R. Jayavel, and F. C. Chou, CrystEngComm 16(37), 8678-8683 (2014).

[54] S. Nie, N. C. Bartelt, J. M. Wofford, O. D. Dubon, K. F. McCarty, and K. Thürmer, Phys. Rev. B 85(20), 205406 (2012).

[55] R. C. Jaklevic and L. Elie, Phys. Rev. Lett. 60(Jan), 120-123 (1988).

[56] J. V. Barth, H. Brune, G. Ertl, and R. J. Behm, Phys. Rev. B 42(Nov), 9307-9318 (1990).

[57] P. Nemes-Incze, Z. Osváth, K. Kamarás, and L. Biró, Carbon 46(11), 1435-1442 (2008).

[58] M. K. Tran, J. Levallois, P. Lerch, J. Teyssier, A. B. Kuzmenko, G. Autès, O. V. Yazyev, A. Ubaldini, E. Giannini, D. van der Marel, and A. Akrap, Phyśs. Rev. Lett. 112(4), 047402 (2014).

[59] V. Gnezdilov, P. Lemmens, D. Wulferding, A. Möller, P. Recher, H. Berger, R. Sankar, and F. C. Chou, Phys. Rev. B 89(May), 195117 (2014). 